\title{
Increase in risk and saving behavior
}

\author{
Larry Y. Tzeng ${ }^{\mathrm{a}, *}$, Jen-Hung Wang ${ }^{\mathrm{b}}$ \\ ${ }^{a}$ Department of Finance, National Taiwan University, 50, Lane 144, Sec. 4, Keelung Road, Taipei, Taiwan \\ ${ }^{\mathrm{b}}$ Department of Finance, Shih Hsin University, Taiwan
}

Received 26 November 2001; received in revised form 19 November 2003; accepted 6 January 2004

\begin{abstract}
This paper extends the traditional unambiguous comparative statics analysis of an increase in risk into the case where individual's utility is a function of two goods rather than of a payoff only. Specifically, we use saving behavior to demonstrate the application of the extension. We find that a first-order stochastic dominance of rate of return causes a nonsatiable and risk-averse borrower to increase his borrowing. A mean-preserving second-order stochastic dominance of rate of return causes a borrower, who is risk-averse and prudent, to decrease his borrowing. Furthermore, we find that a stronger central risk dominance leads a nonsatiable and risk-averse lender to decrease his saving. Last, for the mean-preserving CDF shifts, we give a necessary and sufficient condition for all risk-averse lenders (respectively, borrowers) to decrease their saving (respectively, borrowing).
\end{abstract}

(C) 2004 Elsevier Inc. All rights reserved.

JEL classification: D81; D91

Keywords: Increase in risk; Saving; Stochastic dominance; Central risk dominance

\section{Introduction}

It is well-known that a risk-averse decision maker may not invest less on risky assets when faced with an increase in risk (Rothschild \& Stiglitz, 1970, 1971). To generate determinate comparative statics with respect to an increase in risk, some researchers consider imposing certain constraints on individuals' preferences (Briys, Dionne, \& Eeckhoudt, 1989; Diamond \& Stiglitz, 1974; Dionne \& Eeckhoudt, 1987; Dreze \& Modigliani, 1972), while others imposing restrictions on the increase in risk to get sufficient and/or necessary condi-

\footnotetext{
* Corresponding author.

E-mail address: tzeng@ntu.edu.tw (L.Y. Tzeng).
} 
tions (Black \& Bulkley, 1989; Eeckhoudt \& Hansen, 1980, 1983; Gollier, 1995; Meyer \& Ormiston, 1983, 1985).

Indeed, the unambiguous comparative statics is one of the most important questions in the economic theory of rational decision under uncertainty. The standard model in the literature has been considering cases where there is one choice variable, one source of risk, and the utility function is a function of a single variable (wealth) rather than multiple variables (Katz, 1981; Kraus, 1979, etc.). For this standard model, it is well-known, since the remarkable paper of Meyer and Ormiston (1983), that the determinate comparative statics results are an interplay between three kinds of factors: the restrictions on risk preference, the changes in risk, and the ordinal preferences over certain prospects. Thus, as Gollier (1995) pointed out retrospectively, three strategies can be adopted to generate interesting comparative statics results: First, one can try to restrict the ordinal preferences over certain prospects, which is represented by the payoff function (e.g., Meyer \& Ormiston, 1983). Second, one can restrict types of risk preferences (e.g., Hadar \& Seo, 1990; Rothschild \& Stiglitz, 1971). Third, one can try to restrict the changes in risk in question (e.g., Meyer \& Ormiston, 1985; Sandmo, 1970). The last approach is the mostly adopted and culminates in Gollier (1995), who found the necessary and sufficient condition of unambiguous comparative statics results for risk-averse agents under this approach. Gollier requires that the payoff is monotonic in risk. Hau (2001) generalizes Gollier's theorems to include nonmonotonic payoffs.

Although this line of research has generated many ingenious findings, most papers assumed that individual's utility is a function of payoff rather than a function of multiple goods. However, many economic problems fall into the latter classification. One example is saving under uncertainty where individuals consider the tradeoff between current consumption and future consumption when faced with a random investment return. The problem is typically addressed by over-generation models, where the utility function of individuals is assumed to be a function of current consumption and future consumption rather than of a payoff only. For example, Sandmo (1970) showed that an individual with a decreasing absolute temporal risk aversion could increase his savings with an increase in income risk. Dionne, Eeckhoudt, and Briys (1990) extended the model further to analyze both income risk and capital risk.

This paper intends to integrate traditional models into the case where individual's utility is a function of two goods rather than of a payoff only and fits in the third approach proposed by Gollier. We use the saving behavior modeled by Dionne et al. (1990) to demonstrate the application of this extension. Although the analysis of the paper focuses on individual's saving behavior under uncertainty of the interest rate, the model can be extended for other research. We then follow the methodology derived by Rothschild and Stiglitz (1970) and Gollier (1995) to obtain conditions for unambiguous comparative statics of risk increases.

\section{The model}

Let us assume that an individual needs to make a decision on current consumption, $C_{1}$, and future consumption, $C_{2}$. The individual's (exogenous) wealth in each period is designated as $W_{1}$ and $W_{2}$, respectively. The utility function of the individual is assumed to be additively separable on $C_{1}$ and $C_{2}$, and can be expressed as $U\left(C_{1}, C_{2}\right)=u\left(C_{1}\right)+\beta u\left(C_{2}\right)$, where 
$u(\cdot)$ is an underlying time-invariant von Neumann-Morgenstern utility function and $\beta$ is the time-discount rate for utility. In general, $u(\cdot)$ may have no other specific properties. But we will assume that it is continuous and differentiable as many times as needed. We further assume that the individual can borrow or lend his money by an random interest rate. $r \in[a, b]$, which follows a distribution function $F(r)$. While the randomness of an interest rate seems inadequate for deposit saving, where individuals usually know the interest rate for their deposit, one can interpret the random interest rate as the rate of return of a "saving technology" in a general sense to include both risk-free and risky investments. "Saving" may then present an investment on an uncertain production project or on risky assets; "borrowing" then means short selling such opportunities. When the investment is risk-free, $F(r)$ will be a degenerated distribution.

The individual's budget constraint between two periods can be expressed as

$$
C_{2}=\left(W_{1}-C_{1}\right)(1+r)+W_{2} .
$$

In Eq. (1), $\left(W_{1}-C_{1}\right)$ is considered to be the "saving" of the individual; it can be negative if the individual in fact "borrows". However, both $C_{1}$ and $C_{2}$ must be nonnegative. As stated, the return of individual's saving in Eq. (1) is assumed to be under uncertainty. Such cases could happen when, say, the individual invests his money on risky assets, such as stocks.

Under the expected-utility framework, the individual chooses an optimal current consumption to maximize his expected utility, $\mathrm{EU}\left(C_{1}, C_{2}\right)$. The problem can be written as

$$
\begin{array}{ll}
\operatorname{Max}_{C_{1}} & H \equiv \mathrm{EU}=u\left(C_{1}\right)+\beta \int_{a}^{b} u\left[\left(W_{1}-C_{1}\right)(1+r)+W_{2}\right] f(r) \mathrm{d} r \\
\text { s.t. } & 0 \leq C_{1} \leq \bar{C} \equiv \frac{W_{2}}{1+r}+W_{1}
\end{array}
$$

Assume that the second-order condition of Eq. (2) holds and that an interior solution exists. ${ }^{1}$ The first-order condition of Eq. (2) is

$$
H^{\prime}=u^{\prime}\left(C_{1 f}^{\prime}\right)-\beta \int_{a}^{b}(1+r) u^{\prime}\left[\left(W_{1}-C_{1 f}^{\prime}\right)(1+r)+W_{2}\right] f(r) \mathrm{d} r=0,
$$

where $C_{1 f}^{\prime}$ denotes the optimal current consumption, given the distribution $F(r)$.

Suppose the distribution of the interest rate is shifted from $F(r)$ to $G(r)$ within the same domain $r \in[a, b]$, and let $f(r)$ and $g(r)$ be, respectively, the probability density function of $F(r)$ and $G(r)$. For a nonsatiable, risk-averse borrower, the following theorem gives a sufficient condition on the CDF shifts of rate of return to guarantee that he will borrow more.

Theorem 1. For a net borrower (under the original interest rate distribution $F(r)$ ) who is nonsatiable and risk averse, that is, given that $W_{1}<C_{1 f}^{*}, u^{\prime}>0$ and $u^{\prime \prime}<0$, when the interest rate distribution shifts from $F(r)$ to $G(r)$, he will increase his borrowing (or formally, $\left.C_{1 g}^{\prime} \geq C_{1 f}^{\prime}\right)$, if $G(r) \geq F(r), \forall r \in[a, b]$.

\footnotetext{
${ }^{1}$ Since the opportunity set is compact and the objective function is continuous, this maximization problem always has solution(s). However, we still need to make these assumptions.
} 
Proof of Theorem 1. First rewrite Eq. (3) as the following

$$
H^{\prime}\left(C_{1 f}^{*} ; u, f\right)=\int_{a}^{b} w\left(t, C_{1 f}^{*}, u\right) f(t) \mathrm{d} t=0,
$$

where

$$
w\left(r, C_{1 f}^{*}, u\right)=u^{\prime}\left(C_{1 f}^{\prime}\right)-\beta(1+r) u^{\prime}\left[\left(W_{1}-C_{1 f}^{\prime}\right)(1+r)+W_{2}\right] .
$$

After an integration by parts, Eq. (4) can be rewritten as:

$$
H^{\prime}\left(C_{1 f}^{*} ; u, f\right)=w\left(b, C_{1 f}^{*}, u\right)-\int_{a}^{b} w_{r}\left(t, C_{1 f}^{*}, u\right) F(t) \mathrm{d} t=0,
$$

where

$$
\begin{aligned}
w_{r}\left(r, C_{1 f}^{*}, u\right)= & -\beta u^{\prime}\left[\left(W_{1}-C_{1 f}^{*}\right)(1+r)+W_{2}\right] \\
& -\beta(1+r)\left(W_{1}-C_{1 f}^{*}\right) u^{\prime \prime}\left[\left(W_{1}-C_{1 f}^{*}\right)(1+r)+W_{2}\right] .
\end{aligned}
$$

Since we have $W_{1}<C_{1 f}^{*}, u^{\prime}>0$ and $u^{\prime \prime}<0$, the term $w_{r}\left(r, C_{1 f}^{*}, u\right)$ is definitely negative.

If the distribution is shifted from $F(r)$ to $G(r)$, then

$$
H^{\prime}\left(C_{1 f}^{*} ; u, g\right)=w\left(b, C_{1 f}^{\prime}, u\right)-\int_{a}^{b} w_{r}\left(t, C_{1 f}^{*}, u\right) G(t) \mathrm{d} t .
$$

Thus,

$$
H^{\prime}\left(C_{1 f}^{*} ; u, g\right)-H^{\prime}\left(C_{1 f}^{*} ; u, f\right)=\int_{a}^{b} w_{r}\left(t, C_{1 f}^{*}, u\right)[F(t)-G(t)] \mathrm{d} t .
$$

Given the condition that

$$
G(r) \geq F(r), \forall r \in[a, b],
$$

and recalling that $w_{r}\left(r, C_{1 f}^{*}, u\right)<0, \forall r$ given the conditions $W_{1}<C_{1 f}^{*}, u^{\prime}>0, u^{\prime \prime}<0$, we have

$$
H^{\prime}\left(C_{1 f}^{*} ; u, g\right)-H^{\prime}\left(C_{1 f}^{*} ; u, f\right)=\int_{a}^{b} w_{r}\left(t, C_{1 f}^{*}, u\right)[F(t)-G(t)] \mathrm{d} t \geq 0 .
$$

As $H^{\prime}\left(C_{1 f}^{*} ; u, f\right)=0$, Eq. (9), indeed, implies $H^{\prime}\left(C_{1 f}^{*} ; u, g\right) \geq 0$.

Let $C_{1 g}^{*}$ be the optimal solution of $H^{\prime}\left(C_{1 g}^{*} ; u, g\right)=0$. Immediately, $C_{1 g}^{*} \geq C_{1 f}^{*}$.

Remark. Eq. (8) is a defining property of the first-order stochastic dominance (Hadar \& Russell, 1969).

When $G(r)$ is first-order stochastic dominated by $F(r)$, for any given level of the interest rate $r \in[a, b]$, the probability that the ex-post interest rate exceeding this level is always larger under $F(r)$ than that under $G(r)$. Theorem 1 shows that, for a nonsatiable and risk-averse borrower, who borrows under the original interest rate distribution $F(r)$, if he 
faces a shift of interest rate from $F(r)$ to $G(r)$, knowing that the probability that the ex-post interest rate exceeding any level is becoming smaller than before, he will borrow more. However, let it be noted that under the same CDF shifts, (i) a nonsatiable but risk-loving borrower does not necessarily borrow less (since the corresponding $w_{r}\left(r, C_{1 f}^{*}, u\right)$ for such agent cannot be definitely signed); also (ii) a nonsatiable, risk-averse lender does not necessarily lend less (for the same reason).

In Theorem 1 we find a sufficient condition of unambiguous comparative statics for a nonsatiable and risk-averse borrower to borrow more. By the same methodology, we can further explore a sufficient condition of unambiguous comparative statics for a risk-averse and prudent borrower to borrow less. The result is given in Theorem 2. We first introduce some notation: let $S_{f}(r)$ and $S_{g}(r)$ denote the integration function of $F(r)$ and $G(r)$, respectively, and let $\mu_{f}$ and $\mu_{g}$ denote the mean of $r$ under $F(r)$ and $G(r)$, respectively.

Theorem 2. For a net borrower (under the original interest rate distribution $F(r)$ ) who is risk averse and prudent, that is, given that $W_{1}<C_{1 f}^{*}, u^{\prime \prime}<0$ and $u^{\prime \prime \prime}>0$, when the interest rate distribution shifts from $F(r)$ to $G(r)$, he will decrease his borrowing (or formally, $\left.C_{1 g}^{*} \leq C_{1 f}^{*}\right)$, if $\mu_{f}=\mu_{g}$ and $S_{g}(r) \geq S_{f}(r), \forall r \in[a, b]$.

Proof of Theorem 2. After an integration by parts again, Eq. (5) can be rewritten as

$$
\begin{aligned}
H^{\prime}\left(C_{1 f}^{*} ; u, f\right)= & w\left(b, C_{1 f}^{*}, u\right)-w_{r}\left(b, C_{1 f}^{*}, u\right) S_{f}(b) \\
& +\int_{a}^{b} w_{r r}\left(t, C_{1 f}^{*}, u\right) S_{f}(t) \mathrm{d} t=0,
\end{aligned}
$$

where

$$
\begin{aligned}
w_{r r}\left(r, C_{1 f}^{*}, u\right)= & -2 \beta\left(W_{1}-C_{1 f}^{*}\right) u^{\prime \prime}\left[\left(W_{1}-C_{1 f}^{\prime}\right)(1+r)+W_{2}\right] \\
& -\beta(1+r)\left(W_{1}-C_{1 f}^{\prime}\right)^{2} u^{\prime \prime \prime}\left[\left(W_{1}-C_{1 f}^{*}\right)(1+r)+W_{2}\right] .
\end{aligned}
$$

Note that given the conditions $W_{1}<C_{1 f}^{*}, u^{\prime \prime}<0$, and $u^{\prime \prime \prime}$, the term $w_{r r}\left(r, C_{1 f}^{*}, u\right)$ can be definitely signed to be negative.

If the distribution is shifted from $F(r)$ to $G(r)$, then

$$
H^{\prime}\left(C_{1 f}^{*} ; u, g\right)=w\left(b, C_{1 f}^{*}, u\right)-w_{r}\left(b, C_{1 f}^{*}, u\right) S_{g}(b)+\int_{a}^{b} w_{r r}\left(t, C_{1 f}^{*}, u\right) S_{g}(t) \mathrm{d} t .
$$

Thus, if $\mu_{f}=\mu_{g}$, which implies $S_{f}(b)=S_{g}(b)$, then

$$
H^{\prime}\left(C_{1 f}^{*} ; u, g\right)-H^{\prime}\left(C_{1 f}^{*} ; u, f\right)=\int_{a}^{b} w_{r r}\left(t, C_{1 f}^{*}, u\right)\left[S_{g}(t)-S_{f}(t)\right] \mathrm{d} t .
$$

Given the condition that

$$
S_{g}(r) \geq S_{f}(r), \forall r \in[a, b],
$$


and recalling that $w_{r r}\left(r, C_{1 f}^{*}, u\right)<0, \forall r$ given the conditions $W_{1}<C_{1 f}^{*}, u^{\prime}>0, u^{\prime \prime}<0$, we have

$$
H^{\prime}\left(C_{1 f}^{*} ; u, g\right)-H^{\prime}\left(C_{1 f}^{*} ; u, f\right)=\int_{a}^{b} w_{r r}\left(t, C_{1 f}^{*}, u\right)\left[S_{g}(t)-S_{f}(t)\right] \mathrm{d} t \leq 0 .
$$

Since $H^{\prime}\left(C_{1 f}^{*} ; u, f\right)=0$, Eq. (14) indeed implies $H^{\prime}\left(C_{1 f}^{*} ; u, g\right) \leq 0$.

Let $C_{1 g}^{*}$ be the optimal solution of $H^{\prime}\left(C_{1 g}^{*} ; u, g\right)=0$. Immediately, for all individuals with $u^{\prime \prime}<0$ and $u^{\prime \prime \prime}>0, C_{1 g}^{*} \leq C_{1 f}^{*}$.

Remark. Eq. (13) is a defining property of the second-order stochastic dominance (Hadar $\&$ Russell, 1969). Together with the condition that $\mu_{f}=\mu_{g}$, it is also a defining property of an increase in risk, which is precisely a mean-preserving second-order stochastic dominance (Rothschild \& Stiglitz, 1970).

From Theorem 1 we find that a first-order stochastic dominance in interest rate causes a nonsatiable and risk-averse borrower to increase his borrowing, whereas from Theorem 2 we find that a mean-preserving second-order stochastic dominance in interest rate leads a risk-averse and prudent borrower to decrease his borrowing. It is well known that first-order stochastic dominance implies a decrease in the mean, which would be favorable to a borrower. Thus, Theorem 1 seems to indicate that a nonsatiable and risk-averse borrower values the benefit of the decrease in the mean more than the disutility of an increase in risk under first-order stochastic dominance. On the other hand, Theorem 2 shows that if the mean of interest rate is preserved, a borrower does not like to cope marginally with an increase in interest rate risk and, therefore, indeed decreases his borrowing with an increase in risk under a second-order stochastic dominance.

Theorems 1 and 2 give conditions for unambiguous comparative statics results for certain kinds of borrowers (respectively, nonsatiable, risk-averse ones and risk-averse, prudent ones), but say nothing about the lenders. The following theorem gives a sufficient condition on the CDF shifts of rate of return to guarantee that a nonsatiable and risk-averse lender will lend less. To introduce some notation, let $\tau_{f}(r)=\int_{a}^{r}(1+t) f(t) \mathrm{d} t ; \tau_{g}(r)$ is defined accordingly.

Theorem 3. For a net lender (under the original interest rate distribution $(F(r)$ ) who is nonsatiable and risk averse, that is, given that $W_{1}>C_{1 f}^{*}, u^{\prime}>0$, and $u^{\prime \prime}<0$, when the interest rate distribution shifts from $F(r)$ to $G(r)$, he will decrease his saving (or formally, $\left.C_{1 g}^{*} \geq C_{1 f}^{*}\right)$, if $\exists \lambda \in(-\infty, 1] \ni \tau_{g}(r) \leq \lambda \tau_{f}(r), \forall r \in[a, b]$.

Proof of Theorem 3. Recall Eq. (3) as the following

$$
H^{\prime}\left(C_{1 f}^{*} ; u, f\right)=u^{\prime}\left(C_{1 f}^{*}\right)-\beta \int_{a}^{b}(1+r) u^{\prime}\left[\left(W_{1}-C_{1 f}^{*}\right)(1+r)+W_{2}\right] f(r) \mathrm{d} r=0 .
$$

After an integration by parts, Eq. (15) can be rewritten as:

$$
\begin{aligned}
H^{\prime}\left(C_{1 f}^{*} ; u, f\right)= & u^{\prime}\left(C_{1 f}^{*}\right)-\beta\left\{\tau_{f}(b) u^{\prime}\left[\left(W_{1}-C_{1 f}^{*}\right)(1+b)+W_{2}\right]-\int_{a}^{b}\left(W_{1}\right.\right. \\
& \left.\left.\left.-C_{1 f}^{*}\right) u^{\prime \prime}\left[\left(W_{1}-C_{1 f}^{*}\right)(1+r)+W_{2}\right)\right] \tau_{f}(r) \mathrm{d} r\right\}=0,
\end{aligned}
$$


where, as stated,

$$
\tau_{f}(r)=\int_{a}^{r}(1+t) f(t) \mathrm{d} t .
$$

If the distribution is shifted from $F(r)$ to $G(r)$, then, by similar derivation as that in Eq. (16), we have

$$
\begin{aligned}
H^{\prime}\left(C_{1 f}^{*} ; u, g\right)= & u^{\prime}\left(C_{1 f}^{*}\right)-\beta\left\{\tau_{g}(b) u^{\prime}\left[\left(W_{1}-C_{1 f}^{*}\right)(1+b)+W_{2}\right]\right. \\
& \left.-\int_{a}^{b}\left(W_{1}-C_{1 f}^{*}\right) u^{\prime \prime}\left[\left(W_{1}-C_{1 f}^{*}\right)(1+r)+W_{2}\right)\right] \tau_{g}(r) \mathrm{d} r,
\end{aligned}
$$

where

$$
\tau_{g}(r)=\int_{a}^{r}(1+t) g(t) \mathrm{d} t
$$

Thus, noticing that $H^{\prime}\left(C_{1 f}^{*} ; u, g\right)=0$, we have

$$
\begin{aligned}
H^{\prime}\left(C_{1 f}^{*} ; u, g\right)= & H^{\prime}\left(C_{1 f}^{*} ; u, g\right)-\lambda H^{\prime}\left(C_{1 f}^{*} ; u, f\right) \\
= & u^{\prime}\left(C_{1 f}^{*}\right)(1-\lambda)-\beta u^{\prime}\left[\left(W_{1}-C_{1 f}^{*}\right)(1+b)+W_{2}\right]\left[\tau_{g}(b)-\lambda \tau_{f}(b)\right] \\
& +\beta\left(W_{1}-C_{1 f}^{*}\right) \int_{a}^{b} u^{\prime \prime}\left[\left(W_{1}-C_{1 f}^{*}\right)(1+r)+W_{2}\right]\left[\tau_{g}(r)-\lambda \tau_{f}(r)\right] \mathrm{d} r .
\end{aligned}
$$

If $W_{1}>C_{1 f}^{*}$ and $\exists \lambda \in(-\infty, 1] \ni \tau_{g}(r) \leq \lambda \tau_{f}(r), \forall r$, then $H^{\prime}\left(C_{1 f}^{*} ; u, g\right) \geq 0$ for all individuals with $u^{\prime}>0$ and $u^{\prime \prime}<0$. Immediately, $C_{1 g}^{*} \geq C_{1 f}^{*}$.

The condition of Theorem 3 involves a gross-interest-rate-weighted probability mass function, $\tau_{f}(r)=\int_{a}^{r}(1+t) f(t) \mathrm{d} t$. To get some feeling about this function, one can notice that, after an intergration by parts,

$$
\tau_{f}(r)=\int_{a}^{r}(1+t) f(t) \mathrm{d} t=(1+r) F(r)-\int_{a}^{r} F(t) \mathrm{d} t .
$$

For models where the utility is a function of a payoff, Gollier (1995) finds the least constraint (necessary and sufficient) condition for unambiguous comparative statics when the agents are nonsatiable and risk averse. Gollier's condition, $\exists \lambda \in \Re \ni \tau_{g}(r) \geq \lambda \tau_{f}(r), \forall r$, involves a marginal-payoff-weighted probability mass function $\tau(r)$, which, though denoted by the same symbol $\tau$ as ours here, is parallel to but not exactly identically defined as ours (which deals with utility functions of two consumption goods). Particularly, when the payoff function is linear, Gollier's condition gives one definition of risk dominance, named by Gollier as central risk dominance. It is obvious that, mutatis mutandis, our condition in Theorem 3 is a subset of that of Gollier's. Thus, Theorem 3 shows that a more stringent central risk dominance leads a nonsatiable and risk-averse lender to decrease his saving.

Theorem 3 gives a sufficient condition. To explore this problem more deeply, in the following let us focus on cases where the mean of the random rate of return is preserved. 
When the two distributions compared have the same mean, the attention can be more concentrated on the effect of a change of risk. Due to this concentration, the mean-preserving cases are emphatically investigated in the literature, for example, Rothschild and Stiglitz (1970) and Meyer and Ormiston (1983, 1985).

In the mean-preserving cases, we can get necessary and sufficient conditions for both risk-averse lenders and borrowers, whether they are nonsatiable or not. The following two theorems give these results.

Theorem 4. For any net lender (under the original interest rate distribution $F(r)$ ) who is risk averse, that is, given that $W_{1}>C_{1 f}^{*}$ and $u^{\prime \prime}<0$, when the interest rate distribution shifts from $F(r)$ to a mean-preserving $G(r)$, he will decrease his saving (or formally, $C_{1 g}^{*} \geq C_{1 f}^{*}$ ), if and only if $\tau_{g}(r) \leq \tau_{f}(r), \forall r \in[a, b]$.

Proof of Theorem 4. Sufficiency part: Under the requirement that $F(r)$ and $G(r)$ have the same mean, we have $\tau_{g}(b)=\tau_{f}(b)$. Thus, recalling Eq. (20), and choosing $\lambda=1$, we have

$$
\begin{aligned}
H^{\prime}\left(C_{1 f}^{*} ; u, g\right) & =H^{\prime}\left(C_{1 f}^{*} ; u, g\right)-H^{\prime}\left(C_{1 f}^{*} ; u, f\right) \\
& =\beta\left(W_{1}-C_{1 f}^{*}\right) \int_{a}^{b} u^{\prime \prime}\left[\left(W_{1}-C_{1 f}^{*}\right)(1+r)+W_{2}\right]\left[\tau_{g}(r)-\tau_{f}(r)\right] \mathrm{d} r .
\end{aligned}
$$

Given $W_{1}>C_{1 f}^{*}$, if $\tau_{g}(r) \leq \tau_{f}(r), \forall r$, then $H^{\prime}\left(C_{1 f}^{*} ; u, g\right) \geq 0$ for all individuals with $u^{\prime \prime}<0$. Immediately, $C_{1 g}^{*} \geq C_{1 f}^{*}$. Let it be noted that the sign of $H^{\prime}\left(C_{1 f}^{*} ; u, g\right)$ can be unambiguously determined disregard the sign of $u^{\prime}$.

Necessity part: It is equivalent to proving the following statement: If $\exists r_{0} \in[a, b] \ni$ $\tau_{g}\left(r_{0}\right)>\tau_{f}\left(r_{0}\right)$, then there exists an utility function with $u^{\prime \prime}<0$, for which $C_{1 g}^{*}<C_{1 f}^{*}$. We will show this equivalent statement by construction:

First partition the range $[a, b]$ into two parts, $\Omega_{-}$and $\Omega_{+}$, where $\tau_{g}(r)>(\leq) \tau_{f}(r)$ for all $r \in \Omega_{+}\left(\Omega_{-}\right)$, respectively. Since by hypothesis $\exists r_{0} \in[a, b] \ni \tau_{g}\left(r_{0}\right)>\tau_{f}\left(r_{0}\right)$, the set $\Omega_{+}$is nonempty, indeed with positive measure under the assumption that $\tau_{f, g}(r)$ are continuous.

We construct a utility function with $u^{\prime \prime}<0$ as follows:

$$
u^{\prime \prime}\left[\left(W_{1}-C_{1 f}^{*}\right)(1+r)+W_{2}\right]=-\varepsilon_{1}, \quad \text { if } r \in \Omega_{-},
$$

and

$$
u^{\prime \prime}\left[\left(W_{1}-C_{1 f}^{*}\right)(1+r)+W_{2}\right]=-\varepsilon_{2}, \quad \text { if } r \in \Omega_{+},
$$

where $\varepsilon_{1}, \varepsilon_{2}$ can be two arbitrary positive numbers.

The constructed utility must satisfy Eq. (16), the first-order condition of the original maximization problem. This is without any question as the form of $u^{\prime}$, though must be consistent with our construction with $u^{\prime \prime}$, has enough freedom. 
With such construction Eq. (22) can be rewritten as

$$
\begin{aligned}
H^{\prime}\left(C_{1 f}^{*} ; u, g\right)= & -\beta\left(W_{1}-C_{1 f}^{*}\right) \\
& \times\left\{\varepsilon_{1} \int_{\Omega_{-}}\left[\tau_{g}(r)-\tau_{f}(r)\right] \mathrm{d} r+\varepsilon_{2} \int_{\Omega_{+}}\left[\tau_{g}(r)-\tau_{f}(r)\right] \mathrm{d} r\right\} .
\end{aligned}
$$

In the right-hand side of Eq. (23), $\beta,\left(W_{1}-C_{1 f}^{*}\right), \varepsilon_{1}$ and $\varepsilon_{2}$ are all positive, while the integral is positive under $\Omega_{+}$and negative under $\Omega_{-}$. As the two intergrals are both of bounded values and that $\Omega_{+}$is with positive measure, it is always possible to choose $\varepsilon_{2}$ sufficient large and $\varepsilon_{1}$ sufficient small such that the total sum is negative, implying that $C_{1 g}^{*}<C_{1 f}^{*}$.

Q.E.D.

Theorem 5. For any net borrower (under the original interest rate distribution $F(r)$ ) who is risk averse, that is, given that $W_{1}<C_{1 f}^{*}$ and $u^{\prime \prime}<0$, when the interest rate distribution shifts from $F(r)$ to a mean-preserving $G(r)$, he will decrease his borrowing (or formally, $\left.C_{1 g}^{*} \leq C_{1 f}^{*}\right)$, if and only if $\tau_{g}(r) \leq \tau_{f}(r), \forall r \in[a, b]$.

Proof of Theorem 5. Sufficiency is obvious by inspecting Eq. (22). The proof of the necessity part is the same as that of Theorem 4 , only that now we have $W_{1}<C_{1 f}^{*}$. Hence by choosing $\varepsilon_{2}$ sufficient large and $\varepsilon_{1}$ sufficient small, which is always possible, we have $H^{\prime}\left(C_{1 f}^{*} ; u, g\right)>0$, implying that $C_{1 g}^{*}>C_{1 f}^{*}$.

\section{Conclusion}

This paper extends the unambiguous comparative statics analysis in an increase in risk into the case where individual's utility depends on two goods. The extension can be applied in many economic problems, such as saving, labor, and insurance. Specifically, we use consumption-saving behavior to demonstrate the application of this extension.

We provide alternative sufficient conditions for unambiguous comparative statics of risk increases for several situations. Specifically, a nonsatiable and risk-averse borrower increases his borrowing under a change in rate of return of first-order stochastic dominance, while a risk-averse and prudent borrower decreases his borrowing under a change in rate of return of mean-preserving second-order stochastic dominance. Furthermore, we find that a nonsatiable and risk-averse lender decreases his saving if the shift of return distribution satisfies a stronger central risk dominance. Last but not least, for the mean-preserving CDF shifts, we give a necessary and sufficient condition for all risk-averse lenders (respectively, borrowers) to decrease their saving (respectively, borrowing).

The findings of this paper are closely related to the literature but somehow different from previous research. This indicates that further research on this issue may provide more insight into understanding the theory of risk.

\section{References}

Black, J., \& Bulkley, G. (1989, February). A ratio criterion for signing the effects of an increase in uncertainty. International Economic Review, 30, 119-130. 
Briys, E., Dionne, G., \& Eeckhoudt, L. (1989, December). More on insurance as a Giffen good. Journal of Risk and Uncertainty, 2, 415-420.

Diamond, P., \& Stiglitz, J. (1974, July). Increase in risk and in risk aversion. Journal of Economic Theory, 8, 333-361.

Dionne, G., \& Eeckhoudt, L. (1987, November). Proportional risk aversion, taxation and labor supply under uncertainty. Journal of Economics, 47, 353-366.

Dionne, G., Eeckhoudt, L., \& Briys, E. (1990). Proportional risk aversion and saving decisions under uncertainty. In Henri Louberge (Ed.), Risk, information, and insurance (pp. 67-87). Boston: Kluwer.

Dreze, J., \& Modigliani, F. (1972, December). Consumption decisions under uncertainty. Journal of Economic Theory, 5, 308-335.

Eeckhoudt, L., \& Hansen, P. (1980, December). Minimum and maximum prices, uncertainty and the theory of the competitive firm. American Economic Review, 70, 1064-1068.

Eeckhoudt, L., \& Hansen, P. (1983, July). Micro-economic applications of marginal changes in risk. European Economic Review, 22, 167-176.

Gollier, C. (1995, August). The comparative statics and changes in risk revisited. Journal of Economic Theory, $66,522-535$.

Hadar, J., \& Russell, W. (1969). Rules of ordering uncertain prospects. American Economic Review, 59(1), $25-34$.

Hadar, J., \& Seo, T. K. (1990, August). The effects of shifts in a return distribution on optimal portfolios. International Economic Review, 31, 721-736.

Hau, A. (2001, June). A general theorem on the comparative statics of changes in risk. The Geneva Papers on Risk and Insurance Theory, 26, 25-41.

Katz, E. (1981, October). A note on a comparative statics theorem for choice under risk. Journal of Economic Theory, 25, 318-319.

Kraus, M. (1979, December). A comparative statics theorem for choice under risk. Journal of Economic Theory, $21,510-517$.

Meyer, J., \& Ormiston, M. B. (1983, October). The comparative statics of cumulative distribution function changes for the class of risk-averse agents. Journal of Economic Theory, 31, 153-169.

Meyer, J., \& Ormiston, M. B. (1985, June). Strong increases in risk and their comparative statics. International Economics Review, 26, 425-437.

Rothschild, M., \& Stiglitz, J. (1970, September). Increasing risk I: A definition. Journal of Economic Theory, 2, 225-243.

Rothschild, M., \& Stiglitz, J. (1971, June). Increasing risk II: Its economic consequences. Journal of Economic Theory, 3, 66-84.

Sandmo, A. (1970, July). The effects of uncertainty of saving decisions. Review of Economic Studies, 37, 353-360. 